\title{
Recent Advances in the Management of Severe Gastrointestinal Dysmotility
}

\author{
Dipesh H Vasant (D) ${ }^{1,2}$ \\ Simon Lal (iD) 2,3 \\ 'Neurogastroenterology Unit, \\ Wythenshawe Hospital, Manchester \\ University NHS Foundation Trust, \\ Manchester, UK; ${ }^{2}$ Division of Diabetes, \\ Endocrinology and Gastroenterology, \\ University of Manchester, Manchester, \\ UK; ${ }^{3}$ Intestinal Failure Unit, Salford Royal \\ NHS Foundation Trust, Salford, UK
}

Correspondence: Dipesh H Vasant Neurogastroenterology Unit, Wythenshawe Hospital, Manchester University NHS Foundation Trust, Manchester, UK

Email dipesh.vasant@manchester.ac.uk

\begin{abstract}
Severe gastrointestinal motility disorders with small bowel involvement continue to pose a major clinical challenge to clinicians, particularly because of the limitations of diagnostic tests and the lack of efficacious treatment options. In this article, we review current understanding and the utility of diagnostic modalities and therapeutic approaches, and describe how their limitations may potentially exacerbate prolonged suffering with debilitating symptoms, diagnostic delays, the risk of iatrogenic harm and increased healthcare utilisation in this group of patients. Moreover, observations from intestinal failure units worldwide suggest that this problem could be set to increase in the future, with reported trends of increasing numbers of patients presenting with nutritional consequences. Unfortunately, until recently, there has been a lack of consensus recommendations and guidance to support clinicians with their management approach. The aim of this narrative review is to summarise recent developments in this field following publication of an international census of experts, and subsequent clinical guidelines, which have emphasized the importance of holistic, multidisciplinary care. This is particularly important in achieving good clinical outcomes and ensuring the appropriate use of artificial nutritional support, in order to prevent iatrogenic harm. We discuss how these recent developments may impact clinical practice by supporting the development of specialised clinical services to deliver optimal care, and highlight areas where further research is needed.
\end{abstract}

Keywords: small bowel dysmotility, chronic intestinal pseudoobstruction, enteric dysmotility

\section{Introduction}

The diagnosis of severe gastrointestinal dysmotility always requires prior investigation to exclude structural gastrointestinal pathology. Thereafter, all of the commonly used classification systems overlap significantly, requiring objective evidence of abnormal gut transit/peristalsis in more than one gastrointestinal region or evidence of abnormal full thickness histopathology, with evidence of small bowel involvement; the latter being of particular importance in clinical practice to identify those at increased risk of nutritional compromise. ${ }^{1-4}$

However, until recently, in the absence of consensus guidelines, the diagnosis and management of severe gastrointestinal motility has remained contentious, and a recent international census of experts in the field has revealed a variation in practice. ${ }^{5}$ Moreover, severe gastrointestinal dysmotility appears to be an increasingly common cause for referral to intestinal failure centres, accounting for up to $18 \%$ of adults requiring long-term parenteral nutrition $(\mathrm{PN})$, with suggestion that the numbers referred for this indication may be increasing. ${ }^{5-9}$ 
The aims of this paper are, therefore, to review current understanding and recent advances on the diagnosis and management of severe gastrointestinal dysmotility, the potential impact of recently published clinical practice guidelines, ${ }^{10}$ and to highlight areas for future research.

\section{Advances in the Diagnosis and Classification of Gastrointestinal Dysmotility}

Patients with severe gastrointestinal motility disorders present with a variety of non-discriminatory symptoms including vomiting, pain, abdominal distension and constipation, which correlate poorly with the results of the diagnostic tools available. ${ }^{4,11}$ Many of these symptoms overlap significantly with more common functional gastrointestinal disorders defined by the Rome criteria. ${ }^{12}$ All of the classification systems for gastrointestinal dysmotility are reliant on various combinations of diagnostic tests, some of which are highly invasive, difficult to interpret, and many of which are not widely available and have a number of pitfalls.

Based on findings from radiological and motility tests, small intestinal motility disorders can be sub-classified into Chronic Intestinal Pseudo-obstruction (CIPO) and Enteric Dysmotility (ED). ${ }^{1,3,4,13,14}$ CIPO is defined as chronic/recurrent obstructive-type symptoms with radiological features of a dilated intestine and the absence of any lumen-occluding lesion. ${ }^{13,14}$ In a recent international survey on severe gastrointestinal dysmotility, in the majority of clinicians' practice, CIPO makes up to $\leq 25 \%$ of new referrals seen. ${ }^{5}$ In contrast to CIPO, ED refers to patients with objective evidence of impaired small bowel contractility on antroduodenal manometry (ADM), but without a dilated intestine on radiological investigations. $^{1,15}$

Whilst the clinical significance of a manometric ED diagnosis remains controversial, there is increasing recognition amongst clinicians that CIPO is a distinct, and prognostically important phenotype, that should be recognized separately in patients with suspected dysmotility. ${ }^{5}$ CIPO has been shown to be associated with worse outcomes including chronic intestinal failure, higher mortality and complications including small intestinal bacterial overgrowth, and the need for surgical interventions. ${ }^{4,16,17}$ Therefore, radiological imaging to evaluate intestinal calibre, flow of contrast and exclude mechanical lesions is essential in the diagnosis. However, in patients with suspected dysmotility and a non-dilated gut, the uncertainties partly relate to the limitations of the tests for small bowel dysmotility (Table 1). All of the investigations need to be interpreted taking into consideration any anti-motility medications such as opioids or anticholinergic drugs, which should be discontinued beforehand if at all possible. ADM, which is considered the gold standard test for small bowel motility, is invasive and time intensive requiring up to 6 hours to complete a stationary test or 24 hours for ambulatory studies, and is therefore often poorly tolerated. Other limitations of ADM include variability in results, difficulty in interpretation, poor correlation with symptoms and histopathology, and apparent limited impact on patient management, as well as lack of availability. $5,13,15,16,18-20$ These difficulties result in ADM being infrequently used, with only $21 \%$ of clinicians with an interest in severe gastrointestinal motility requesting the test in $>50 \%$ of cases. ${ }^{5}$ Wireless motility capsule (WMC), is a minimally invasive test which provides segmental, and whole gut transit times using pressure, $\mathrm{pH}$ and temperature sensors during its transit through the gastrointestinal tract, which can be helpful in evaluating small bowel transit. ${ }^{21-25}$ WMC carries a similar risk of capsule retention to that of video capsule endoscopy, which can be mitigated by use of a prior patency capsule. Unfortunately, this test is not widely in use, contributed to by the high financial costs associated with the capsules, and is only regularly ordered by $17 \%$ of clinicians involved with managing small intestinal dysmotility in a recent international survey. ${ }^{5}$ MRI is an attractive technique to study motility. Indeed, recent data using cine-MRI has shown some promise for characterizing small bowel motility; however, this requires expensive advanced technology and its interpretation requires considerable expertise, and use of specific protocols which are not widely available, such that its utility requires further study. ${ }^{20,26,27}$ For example, a very recent study comparing MRI motility findings after a test meal between eight patients with CIPO and healthy subjects demonstrated abnormal postprandial activation in patients with CIPO, but surprisingly showed a wide variation in the patterns of contractility, with evidence of hyperactive motility in half the patients studied. ${ }^{28}$ The clinical significance of these findings of cine MRI is unclear, and therefore further studies are required.

Another area of controversy in the diagnosis and classification of gastrointestinal dysmotility is the role of full thickness biopsies taken from the jejunum, usually via laparoscopy. In this context, there are international consensus guidelines for histopathological diagnosis of 
Table I Advantages and Disadvantages of Small Bowel Motility Investigations

\begin{tabular}{|c|c|c|}
\hline $\begin{array}{l}\text { Investigation } \\
\text { Modality }\end{array}$ & Advantages & Disadvantages \\
\hline $\begin{array}{l}\text { Antroduodenal } \\
\text { Manometry }\end{array}$ & $\begin{array}{l}\text { - Recognisable patterns of dysmotility can be identified (eg absent migrating motor } \\
\text { complexes, low amplitude contractions, abnormal propagation of antral and } \\
\text { duodenal contractions, absent fed response). } \\
\text { - Can be given prokinetic drugs during test (eg Octreotide, erythromycin) to evoke } \\
\text { motility responses }\end{array}$ & $\begin{array}{l}\text { - Invasive, } \\
\text { - Often requires endoscopic and } \\
\text { - fluoroscopic guided placement } \\
\text { - Time intensive, } \\
\text { - Difficult to interpret, } \\
\text { - Poor correlation with clinical } \\
\text { outcomes, } \\
\text { - Limited availability, } \\
\text { - Often poorly tolerated, } \\
\text { - Operator dependent }\end{array}$ \\
\hline $\begin{array}{l}\text { Wireless } \\
\text { motility capsule }\end{array}$ & $\begin{array}{l}\text { - Easy to perform } \\
\text { - Non invasive } \\
\text { - Provides whole gut and segmental transit times. } \\
\text { - Easy to interpret }\end{array}$ & $\begin{array}{l}\text { - Risk of capsule retention } \\
\text { - Expensive } \\
\text { - Not widely available } \\
\text { - Not able to test role of medications } \\
\text { in evoking motility responses }\end{array}$ \\
\hline Cine MRI & $\begin{array}{l}\text { - Assessment of calibre of bowel as well as motility } \\
\text { - Ability to evoke motility responses with medications during test }\end{array}$ & $\begin{array}{l}\text { - Limited availability } \\
\text { - Expensive technology } \\
\text { - Time intensive } \\
\text { - Requires radiological expertise } \\
\text { - Unsuitable for patients with } \\
\text { claustrophobia } \\
\text { - Would need to be able to tolerate } \\
\text { preparation/test meal }\end{array}$ \\
\hline
\end{tabular}

gastrointestinal neuromuscular disorders (GINMD) $)^{2,29}$ and patients with small intestinal dysmotility have been shown to have a high incidence of GINMD such as visceral myopathy or neuropathy. ${ }^{16,30}$ However, the diagnostic utility has to be balanced with the risks of invasive surgery, ${ }^{3}$ and a recent international survey has shown that only $16 \%$ of specialists with an interest in this field request full thickness biopsies routinely to evaluate patients with putative small intestinal dysmotility, ${ }^{5}$ with the majority reporting that full thickness biopsies seldom influence medical or nutritional management, and surgical decision-making. ${ }^{5}$ This is reflected in recently published guidelines which suggest reserving full thickness biopsies as an "en passant" procedure in patients undergoing surgery for another indication. $^{10}$

Whilst most cases of severe gastrointestinal dysmotility are idiopathic, ${ }^{5}$ it is important to screen for recognised secondary systemic causes of myopathy and neuropathy, which in some cases can result in specific treatments targeting the underlying condition. A number of screening tests for secondary causes including viral serology ${ }^{31}$ and those recommended in the recently published British Society of Gastroenterology (BSG) guidelines on small bowel dysmotility ${ }^{10}$ are summarised in Box 1.

Recently, there has been an increasing awareness of an association between hypermobile Ehlers-Danlos syndrome (hEDS) and disorders of gut-brain interaction, ${ }^{32,33}$ particularly in the U.K. ${ }^{5}$ with these patients representing up to a third of patients seen in tertiary neurogastroenterology clinics often presenting with symptoms of visceral hypersensitivity. These symptoms respond poorly to opioids and anti-emetics such as cyclizine, ${ }^{33,34}$ which are best avoided in this situation due to the risks of dependency. Whilst associations between hEDS, postural orthostatic tachycardia syndrome and segmental dysmotility affecting different parts of the gastrointestinal tract have been demonstrated, ${ }^{34,35}$ there is currently no evidence for any specific treatment approaches, or evidence to suggest a greater degree of small bowel dysmotility in this group of patients. ${ }^{10}$ 
Box I Recommended Screening Tests for Secondary Dysmotility

- Exclude hypothyroidism, coeliac disease and diabetes

- Viral screen: JC virus, herpes simplex virus, Epstein Barr virus, cytomegalovirus, varicella zoster virus

- Chest imaging - exclude thymoma or other neoplasia (eg, small cell carcinoma of lung)

- Antibodies for scleroderma (anti-centromere, anti Sc I70, anti M3R) and other connective tissue disorders (ANA, ANCA, anti DNA, anti SMA)

- Paraneoplastic antibody screen (small cell carcinoma and thymoma).

- Type I anti-neuronal nuclear antibody (ANNA-I "anti Hu")

- Anti-collapsin response mediator protein 5 (anti CRMP-5/anti CV2)

- Ganglionic acetyl cholinesterase receptor antibody (AChR antibody)

- Anti-voltage gated potassium channel (VGKC)-complex antibodies.

- Mitochondrial disorder screen

Plasma and urine thymidine and deoxyuridine,

WBC thymine phosphorylase.

If high suspicion then test TYMP gene and screen for related diseases (eg, mitochondrial encephalopathy with lactic acidosis and stroke-like episodes).

- Muscle biopsy and sequencing of mitochondrial genome may be considered.

- If none positive consider full thickness jejunal biopsy.

Note: Data from Nightingale et al ${ }^{10}$ and Sinagra et al. ${ }^{31}$

In the absence of an identified secondary cause, the BSG guidelines have recommended that a definitive diagnosis can only be given when a detailed history, symptoms and investigations including full thickness biopsy histology are indicative of gastro-intestinal dysmotility. If, as is commonly the case, a definitive diagnosis is not possible, making an empirical working diagnosis of "probable severe dysmotility" has been recommended. ${ }^{10}$

In cases of suspected severe gastrointestinal dysmotility, a variety of segmental motility investigations can be helpful in evaluating for evidence of pan-enteric involvement. CIPO, for example, has been shown to affect other parts of the gastrointestinal tract other than the small intestine in up to $90 \%$ of cases. ${ }^{36}$ Thus, a variety of segmental motility tests are often requested, the most popular of which include gastric emptying scintigraphy, $\mathrm{x}$-ray colonic transit tests, and oesophageal manometry. ${ }^{5}$ A role for combined gastric and small bowel scintigraphy has recently been suggested in patients with foregut/dyspeptic symptoms, ${ }^{37}$ but the utility of this approach in patients with severe gastrointestinal dysmotility is unclear and would require further study in this specific population.
Based upon the evidence summarised above, and the various difficulties highlighted with different investigation modalities, until better diagnostic methods are available, a pragmatic approach is typically beneficial in diagnosing severe gastrointestinal dysmotility. It has been suggested that this should take into consideration the patient's symptom profile, objective evidence of abnormal motility in $>1$ region on segmental investigations, or abnormal GI neuromuscular histopathology (when available), with evidence of small bowel involvement, either abnormal ADM, abnormal small bowel transit test or intolerance of small bowel feeding (Figure 1). ${ }^{3,16}$ Intolerance of small bowel feeding, whilst subjective, and potentially influenced by other factors, can be a useful pragmatic surrogate for small bowel function.

\section{Current Approaches to the Management of Severe Gastrointestinal Dysmotility Pharmacological Therapies}

Pharmacological treatment of primary severe gastrointestinal dysmotility is usually directed at the patient's specific symptoms, with as few drugs as possible and avoiding high doses of opioids. The recent BSG guidelines recommended thatwhere there is an identified secondary cause, treatment can be directed at the underlying condition (eg connective tissue diseases, enteric myositis, neoplastic disease or myotonic dystrophy). ${ }^{10}$ As discussed earlier, clinicians mostly report that full thickness histopathology results rarely change management. ${ }^{5}$ However, when histopathology results detect an inflammatory neuromuscular infiltrate, immunosuppressive therapy can be considered as a targeted therapeutic option in an attempt to reverse the situation. ${ }^{38-41}$ In practice, this is often ineffective, presumably due to the long-term effects of the immune mediated insult on the regulatory cells of the enteric nervous system, interstitial cells of Cajal and smooth muscle. ${ }^{18}$

Overall, drug treatments for gastrointestinal dysmotility have significant limitations and often, despite correcting physiological abnormalities, may not have any positive effect in improving the patients' symptoms. In the recent international survey, the vast majority of clinicians did not rate any medical treatments as being effective for $>50 \%$ of patients. ${ }^{5}$ The drug treatments that ranked best in the survey were antibiotics to treat small intestinal bacterial overgrowth (SIBO), prucalopride, and neuropathic analgesics. $^{5}$ Presumably, as a consequence of 




Figure I An updated evidence-based algorithm for the pragmatic approach to diagnosing and managing severe gastrointestinal dysmotility Notes: Adapted from Paine P, McLaughlin J, Lal S. Review article: the assessment and management of chronic severe gastrointestinal dysmotility in adults. Aliment Pharmacol Ther. 2013;38(I0):1209-1229. ${ }^{3}$ @ 2013 John Wiley \& Sons Ltd.

gastrointestinal stasis, patients with objective small bowel dysmotility have been shown to have a higher incidence of SIBO, ${ }^{24,42}$ particularly in the CIPO sub-type. ${ }^{16}$ Despite their well-documented limitations, ${ }^{43}$ glucose-hydrogen breath tests are frequently used in practice to support an objective diagnosis of $\mathrm{SIBO}^{5}$ although, where there is a strong clinical suspicion of SIBO, an empirical trial of antibiotics is justifiable. ${ }^{43}$ In this context, recent consensus guidelines on the utility of breath tests ${ }^{44}$ and management of SIBO including recommendations on antibiotic treatment have proven to be a useful resource for clinicians. ${ }^{45}$ As also indicated in the recent BSG guidelines, there are relatively few good prokinetic options amidst cautions with the long-term use of domperidone which requires ECG monitoring of QTc intervals, the potential for extrapyramidal effects of long-term metoclopramide, and tachyphylaxis with erythromycin. ${ }^{10}$ However, supporting the results from the recent international survey, there is now an increasing evidence-base for the 5HT4 agonist Prucalopride in gastrointestinal motility disorders as a pangut prokinetic. In addition to its known effects on small bowel and colonic transit, ${ }^{46,47}$ Prucalopride has recently been shown to improve foregut sensorimotor function, ${ }^{48-50}$ and has also previously been shown to have some benefit in improving symptoms in patients with CIPO. ${ }^{51}$ The other group of treatments that were reported to be highly effective in the recent survey was neuropathic analgesics. Chronic abdominal pain is a common, but particularly challenging symptom to treat in gastrointestinal dysmotility, with many of the medical treatments such as anticholinergics having antimotility effects, and opioids being relatively contraindicated due to their deleterious and counterintuitive effects which can often exacerbate the pain secondary to hyperalgesia and development of 
Narcotic Bowel Syndrome. ${ }^{52}$ However, in recent years, there have been advances in the understanding of the gut-brain interactions in the pathophysiology and treatment of chronic gastrointestinal pain, ${ }^{52}$ which have led to recommendations for avoidance of opioids, and the use of centrally acting gut-brain neuromodulators which are associated with more positive outcomes. ${ }^{53-55}$ Other treatments which have been recommended for severe gastrointestinal dysmotility include the somatostatin analogue octreotide and pyridostigmine. ${ }^{10}$ Subcutaneous octreotide (50$100 \mathrm{mcg}$ once to twice daily), via its effects on sensory afferent pathways and direct effects on small bowel motility, may be effective ${ }^{56,57}$ in selected cases. ${ }^{58}$ Similarly, Pyridostigmine, which enhances parasympathetic activity in the gut to increase intestinal motility, ${ }^{59-61}$ may have a role in this setting, ${ }^{62}$ although the specific data to support its use are limited to small case series and case reports. At present, there is no specific evidence base to support the use of probiotics or faecal microbial transplantation in the treatment of severe gastrointestinal motility disorders, and therefore their use is not currently recommended in this setting, but may be the subject of future research studies.

\section{Nutritional Optimisation}

The recent BSG guidelines have emphasised the importance of nutritional assessment in the management of severe gastrointestinal dysmotility. ${ }^{10}$ Dietary modification of food consistency can improve eating ability; these recommendations include the use of oral liquid feeds, increasing the frequency of oral intake, with low-fat, lowfiber, low-lactose, smaller portions, and liquid nutritional supplements as first-line approaches. ${ }^{10,20}$ One of the main goals in gastrointestinal motility disorders is ensuring adequate nutritional status, and dietary education can be sufficient in patients with mild to moderate symptoms. ${ }^{20}$ Patients should be assessed for vitamin and micronutrient deficiencies and appropriate supplementation should be prescribed when necessary. ${ }^{10}$ Nutritional support via enteral tube feeding should be reserved for patients with objective evidence of malnutrition, with clear goals of therapy, rather than to treat symptoms alone, ${ }^{63}$ and enteral tube placement early in the course of illness should be avoided if possible. ${ }^{10}$ Parenteral nutrition should be reserved solely for cases where malnutrition and electrolyte abnormalities persist and cannot be managed safely with oral supplements/adjustments, gastric or jejunal tube feeding. ${ }^{10}$ Most clinicians with experience in intestinal failure report that this group of patients make up $10-25 \%$ of their overall caseload of patients on long-term parenteral nutrition, and that parenteral nutrition seldom improves symptoms, often leads to long-term parenteral feeding dependency, and, when compared to other causes of intestinal failure, higher rates of catheter-related complications and psychological comorbidity, especially within the ED sub-group. ${ }^{5}$ Indeed, the risks associated with long-term parenteral nutrition, some of which can be lifethreatening, should not be underestimated and should be explained in detail to the patient before considering embarking on this modality of feeding. There is certainly increasing awareness of the psychological impact of severe gastrointestinal dysmotility diagnoses ${ }^{64}$ and the roles of psychosocial support, ${ }^{65}$ and access to a clinical psychologist, ${ }^{5,10}$ as part of integrated multidisciplinary care for these patients. Parenteral nutrition can have an important life sustaining role when nutrition, electrolytes, and hydration status cannot be maintained safely by other means. There is relatively recent data from a number of intestinal failure units internationally supporting this with 5-year survival rates on parenteral nutrition of $70-85 \%,{ }^{16,66-68}$ which are comparable to survival rates in patients with short bowel syndrome. ${ }^{6,67}$ It is becoming apparent that parenteral nutrition requirements and longterm dependency are higher in patients with the CIPO subtype, ${ }^{4,16,17}$ therefore underlining the importance of recognising this sub-type separately to guide management.

\section{Endoscopic Management}

The role of endoscopic management in severe gastrointestinal dysmotility is limited. In the context of CIPO specifically, endoscopic decompression of distended gastrointestinal segments can be beneficial, and depending on the response, in selected cases an endoscopically placed "venting" gastrostomy or percutaneous colostomy tube can be considered. ${ }^{18}$

\section{Surgical Management}

In the past, it has been well recognised that patients with severe gastrointestinal dysmotility often have unnecessary surgical interventions, ${ }^{69}$ with a high risk of iatrogenic complications, and limited benefit. ${ }^{13}$ Surgical intervention in this setting has been shown to be associated with a high incidence of morbidity, mortality and re-operation. ${ }^{69}$ It is therefore recommended that surgical interventions should be limited to judicious, palliative interventions such as stoma formation targeting improvement in symptoms and quality of life, although somewhat less invasive approaches such as 
venting gastrostomy placement, where appropriate, should of course be considered first. ${ }^{10,70,71}$

Small bowel transplantation is only currently recommended for patients who develop significant, irreversible, complications of parenteral nutrition including intestinal failure associated liver disease or reduced venous access secondary to extensive thrombosis. ${ }^{10}$ In the recent international survey, three quarters of intestinal failure experts that participated indicated that they would refer patients with severe gastrointestinal dysmotility if they met these criteria. ${ }^{5}$ Data from a recently published series of 55 patients with endstage CIPO and parenteral nutrition associated complications have reported a $69 \% 5$-year survival rate, ${ }^{72}$ with sustained nutritional autonomy in $70 \%$ of those transplanted and improvements in the quality of life of long-term survivors. ${ }^{72}$

\section{Implications of Recent Advances on Clinical Practice and Future Directions}

Despite the recent advances summarised in this article, the diagnosis and management of severe gastrointestinal motility disorders continue to pose significant clinical challenges. Whilst there are limitations of diagnostic tests, there is now increasing evidence for the role of recognising CIPO as a prognostically significant entity in its own right. The diagnosis and management of patients without a dilated intestine is, however, more contentious. There is clearly a need for better diagnostic technologies for assessing small bowel transit but, until then, the pragmatic approach illustrated in Figure 1 can be helpful in clinical practice.

This paper highlights the unmet need for more effective drug treatments. With advances in the understanding of enteric nervous system neurobiology, further research is now required to develop therapies targeting novel neurochemical and hormonal mechanisms to determine if these can improve gastrointestinal transit. In the meantime, the recent BSG clinical guidelines have provided an important framework for clinical practice. In particular, an approach to systematically excluding secondary and iatrogenic causes, and the role of careful interpretation of investigations to exclude mechanical causes and segmental motility tests are particularly important. One of the main highlights of the recent literature in this field is the role of holistic multidisciplinary care in management. As discussed in detail in this paper, specific medical, nutritional, and surgical interventions all have limited impact as stand-alone treatments in symptom control and management of the condition. It is therefore paramount that an holistic approach to patient care is adopted, targeting primary symptoms and correcting malnutrition, within a biopsychosocial framework, a concept now becoming universal within the field of neurogastroenterology.

The international census, clinical guidelines, and recent data from intestinal failure units have now helped define key members of a specialist multidisciplinary team in order to achieve optimal outcomes. An ideal team should comprise sub-specialist input and leadership from clinicians including gastroenterologists with an interest in neurogastroenterology, gastrointestinal physiologists, psychologists, pain management experts, specialist dieticians, radiologists, specialist nurses and histopathologists with an interest in gastrointestinal neuromuscular disorders.

Opioid use and psychological factors are ranked by clinicians as two of the top three most important predictive factors for long-term parenteral nutrition dependency, ${ }^{5}$ and therefore are two potentially modifiable factors which should be addressed in clinical practice. Often patients suffer with chronic pain, and are prescribed long-term opioid analgesia which can be significantly detrimental, not least due to their antimotility effects, and often make the pain worse due to opioid induced hyperalgesia. ${ }^{53,73}$ Therefore, access to a specialist pain management team to assist with weaning from opioids and implement more appropriate, alternative pain management strategies is extremely beneficial within the multidisciplinary team. Furthermore, the role of the clinical psychologist within gastroenterology is being increasingly recognised, ${ }^{74}$ especially within neurogastroenterology, ${ }^{75}$ with psychogastroenterology rapidly emerging as a subspecialty within the field in its own right. Indeed, psychological treatments were ranked by clinicians in the recent international survey as the second most effective treatment modality overall in severe gastrointestinal dysmotility. ${ }^{5}$ A range of different psychological interventions including gut-focused hypnotherapy ${ }^{76}$ can be beneficial in patients with severe gastrointestinal motility disorders. ${ }^{16}$

Due to the clinical complexities outlined in this article, even within the auspices of specialist multidisciplinary teams, management can be challenging but, within this integrated approach, patients can be treated holistically and efficiently, to provide the best possible chance of avoiding complications and harm, optimising quality of life, and the best chances of nutritional autonomy. 


\section{Conclusions}

Patients with suspected severe gastrointestinal dysmotility require holistic, specialist integrated care, within a biopsychosocial framework in a multidisciplinary setting. The CIPO sub-type appears to be associated with a worse prognosis and should be recognised as a separate entity to ED. The emphasis of care should be on earlier recognition and diagnosis, targeting symptoms with medical and non-medical approaches, treating complications including suspected small intestinal bacterial overgrowth, and safely optimising nutrition and hydration status with the least invasive option possible.

\section{Disclosure}

Neither author has any relevant conflicts of interest to declare for this work.

\section{References}

1. Wingate D, Hongo M, Kellow J, Lindberg G, Smout A. Disorders of gastrointestinal motility: towards a new classification. $J$ Gastroenterol Hepatol. 2002;17(Suppl):S1-14. doi:10.1046/ j.1440-1746.17.s1.7.x

2. Knowles CH, De Giorgio R, Kapur RP, et al. The London classification of gastrointestinal neuromuscular pathology: report on behalf of the Gastro 2009 International Working Group. Gut. 2010;59 (7):882-887. doi:10.1136/gut.2009.200444

3. Paine P, McLaughlin J, Lal S. Review article: the assessment and management of chronic severe gastrointestinal dysmotility in adults. Aliment Pharmacol Ther. 2013;38(10):1209-1229. doi:10.1111/ apt. 12496

4. Lindberg G, Iwarzon M, Tornblom H. Clinical features and long-term survival in chronic intestinal pseudo-obstruction and enteric dysmotility. Scand $J$ Gastroenterol. 2009;44(6):692-699. doi:10.1080/00365520902839642

5. Vasant DH, Pironi L, Barbara G, et al. An international survey on clinicians' perspectives on the diagnosis and management of chronic intestinal pseudo-obstruction and enteric dysmotility. Neurogastroenterol Motil. 2020;32(12):e13937. doi:10.1111/ nmo. 13937

6. Dibb M, Soop M, Teubner A, et al. Survival and nutritional dependence on home parenteral nutrition: three decades of experience from a single referral centre. Clin Nutr. 2017;36(2):570-576. doi:10.1016/ j.clnu.2016.01.028

7. Smith T, Hirst A, Jones B, Baxter J. Annual BANS report. British Association of Parenteral and Enteral Nutrition; 2011.

8. Pironi L, Arends J, Bozzetti F, et al. ESPEN guidelines on chronic intestinal failure in adults. Clin Nutr. 2016;35(2):247-307.

9. Pironi L, Joly F, Forbes A, et al. Long-term follow-up of patients on home parenteral nutrition in Europe: implications for intestinal transplantation. Gut. 2011;60(1):17-25. doi:10.1136/gut.2010.223255

10. Nightingale JM, Paine P, McLaughlin J, Emmanuel A, Martin JE, Lal S. The management of adult patients with severe chronic small intestinal dysmotility. Gut. 2020;69(12):2074-2092. doi:10.1136/ gutjnl-2020-321631

11. Mann SD, Debinski HS, Kamm MA. Clinical characteristics of chronic idiopathic intestinal pseudo-obstruction in adults. Gut. 1997;41(5):675-681. doi:10.1136/gut.41.5.675

12. Drossman DA, Hasler WL. Rome IV-functional GI disorders: disorders of Gut-brain interaction. Gastroenterology. 2016;150 (6):1257-1261. doi:10.1053/j.gastro.2016.03.035
13. Stanghellini V, Cogliandro RF, De Giorgio R, et al. Natural history of intestinal failure induced by chronic idiopathic intestinal pseudo-obstruction. Transplant Proc. 2010;42(1):15-18. doi:10.1016/j.transproceed.2009.12.017

14. Camilleri M. Intestinal dysmotility: does the X-ray resolve the real dilemma? J Pediatr Gastroenterol Nutr. 1997;24(1):100-101. doi:10.1097/00005176-199701000-00020

15. Quigley EM. Enteric dysmotility: validating the Wingate/Bangkok classification. Gastroenterology. 2010;139(1):346-348. doi:10.1053/j. gastro.2010.05.031

16. Vasant DH, Kalaiselvan $\mathrm{R}$, Ablett $\mathrm{J}$, et al. The chronic intestinal pseudo-obstruction subtype has prognostic significance in patients with severe gastrointestinal dysmotility related intestinal failure. Clin Nutr. 2018;37(6):1967-1975. doi:10.1016/j.clnu.2018.09.008

17. Elfadil O, Velapati S, Hurt R, Lal S, Mundi M. Utility of sub-classification of severe gastrointestinal dysmotility in chronic intestinal failure. Clin Nutr ESPEN. 2020;40:531. doi:10.1016/j. clnesp.2020.09.373

18. Di Nardo G, Di Lorenzo C, Lauro A, et al. Chronic intestinal pseudo-obstruction in children and adults: diagnosis and therapeutic options. Neurogastroenterol Motil. 2017;29(1).

19. Malagelada C, Karunaratne TB, Accarino A, et al. Comparison between small bowel manometric patterns and full-thickness biopsy histopathology in severe intestinal dysmotility. Neurogastroenterol Motil. 2017.

20. Billiauws L, Corcos O, Joly F. Dysmotility disorders: a nutritional approach. Curr Opin Clin Nutr Metab Care. 2014;17(5):483-488. doi:10.1097/MCO.0000000000000095

21. Farmer AD, Scott SM, Hobson AR. Gastrointestinal motility revisited: the wireless motility capsule. United European Gastroenterol $J$. 2013;1(6):413-421. doi:10.1177/2050640613510161

22. Rao SS, Camilleri M, Hasler WL, et al. Evaluation of gastrointestinal transit in clinical practice: position paper of the American and European Neurogastroenterology and Motility Societies. Neurogastroenterol Motil. 2011;23(1):8-23. doi:10.1111/j.13652982.2010.01612.x

23. Rao SS, Mysore K, Attaluri A, Valestin J. Diagnostic utility of wireless motility capsule in gastrointestinal dysmotility. J Clin Gastroenterol. 2011;45(8):684-690. doi:10.1097/ MCG.0b013e3181ff0122

24. Roland BC, Ciarleglio MM, Clarke JO, et al. Small intestinal transit time is delayed in small intestinal bacterial overgrowth. $J$ Clin Gastroenterol. 2015;49(7):571-576. doi:10.1097/ MCG.0000000000000257

25. Farmer AD, Wegeberg AL, Brock B, et al. Regional gastrointestinal contractility parameters using the wireless motility capsule: inter-observer reproducibility and influence of age, gender and study country. Aliment Pharmacol Ther. 2018;47(3):391-400. doi:10.1111/apt.14438

26. Fuyuki A, Ohkubo H, Higurashi T, et al. Clinical importance of cine-MRI assessment of small bowel motility in patients with chronic intestinal pseudo-obstruction: a retrospective study of 33 patients. $J$ Gastroenterol. 2017;52(5):577-584. doi:10.1007/s00535-0161251-8

27. Menys A, Butt S, Emmanuel A, et al. Comparative quantitative assessment of global small bowel motility using magnetic resonance imaging in chronic intestinal pseudo-obstruction and healthy controls. Neurogastroenterol Motil. 2016;28(3):376-383. doi:10.1111/ nmo. 12735

28. van Rijn KL, Bredenoord AJ, Smout AJPM, et al. Fasted and fed small bowel motility patterns at cine-MRI in chronic intestinal pseudo-obstruction. Neurogastroenterol Motil. 2020:e14062.

29. Knowles CH, Veress B, Tornblom H, et al. Safety and diagnostic yield of laparoscopically assisted full-thickness bowel biospy. Neurogastroenterol Motil. 2008;20(7):774-779. doi:10.1111/j.13652982.2008.01099.x 
30. Lindberg G, Tornblom H, Iwarzon M, Nyberg B, Martin JE, Veress B. Full-thickness biopsy findings in chronic intestinal pseudo-obstruction and enteric dysmotility. Gut. 2009;58 (8):1084-1090. doi:10.1136/gut.2008.148296

31. Sinagra E, Pellegatta G, Maida M, et al. Could chronic idiopatic intestinal pseudo-obstruction be related to viral infections? J Clin Med. 2021;10(2):268. doi:10.3390/jcm10020268

32. Lam CY, Palsson OS, Whitehead WE, et al. Rome IV functional gastrointestinal disorders and health impairment in subjects with hypermobility spectrum disorders or hypermobile Ehlers-Danlos Syndrome. Clin Gastroenterol Hepatol. 2020. doi:10.1016/j. cgh.2020.02.034

33. Fikree A, Aktar R, Grahame R, et al. Functional gastrointestinal disorders are associated with the joint hypermobility syndrome in secondary care: a case-control study. Neurogastroenterol Motil. 2015;27(4):569-579. doi:10.1111/nmo.12535

34. Beckers AB, Keszthelyi D, Fikree A, et al. Gastrointestinal disorders in joint hypermobility syndrome/Ehlers-Danlos syndrome hypermobility type: a review for the gastroenterologist. Neurogastroenterol Motil. 2017;29(8):e13013. doi:10.1111/nmo.13013

35. Alomari M, Hitawala A, Chadalavada P, et al. Prevalence and predictors of gastrointestinal dysmotility in patients with hypermobile Ehlers-Danlos syndrome: a tertiary care center experience. Cureus. 2020;12(4).

36. Amiot A, Joly F, Cazals-Hatem D, et al. Prognostic yield of esophageal manometry in chronic intestinal pseudo-obstruction: a retrospective cohort of 116 adult patients. Neurogastroenterol Motil. 2012;24 (11):1008-e1542. doi:10.1111/j.1365-2982.2012.01973.x

37. Maurer $\mathrm{AH}, \mathrm{Yu} \mathrm{D}, \mathrm{Lu} \mathrm{X}$, et al. Addition of small-bowel transit scintigraphy to gastric emptying for assessment of patients with upper gastrointestinal symptoms. Neurogastroenterol Motil. 2021;33 (2):e13987. doi:10.1111/nmo.13987

38. Schäppi M, Smith V, Milla P, Lindley K. Eosinophilic myenteric ganglionitis is associated with functional intestinal obstruction. Gut. 2003;52(5):752-755. doi:10.1136/gut.52.5.752

39. Ooms AH, Verheij J, Hulst JM, et al. Eosinophilic myenteric ganglionitis as a cause of chronic intestinal pseudo-obstruction. Virchows Archiv. 2012;460(1):123-127. doi:10.1007/s00428-011-1183-x

40. Oton E, Moreira V, Redondo C, et al. Chronic intestinal pseudo-obstruction due to lymphocytic leiomyositis: is there a place for immunomodulatory therapy? Gut. 2005;54(9):1343-1344. doi:10.1136/gut.2005.071811

41. Dewit S, De Hertogh G, Geboes K, Tack J. Chronic intestinal pseudo-obstruction caused by an intestinal inflammatory myopathy: case report and review of the literature. Neurogastroenterol Motil. 2008;20(4):343-348. doi:10.1111/j.1365-2982.2007.01033.x

42. Jacobs C, Coss Adame E, Attaluri A, Valestin J, Rao SSC. Dysmotility and proton pump inhibitor use are independent risk factors for small intestinal bacterial and/or fungal overgrowth. Aliment Pharmacol Ther. 2013;37(11):1103-1111. doi:10.1111/apt.12304

43. Arasaradnam RP, Brown S, Forbes A, et al. Guidelines for the investigation of chronic diarrhoea in adults: British Society of Gastroenterology. Gut. 2018;67(8):1380-1399. doi:10.1136/gutjnl2017-315909

44. Rezaie A, Buresi M, Lembo A, et al. Hydrogen and methane-based breath testing in gastrointestinal disorders: the North American consensus. $\mathrm{Am}$ J Gastroenterol. 2017;112(5):775. doi:10.1038/ajg.2017.46

45. Pimentel M, Saad RJ, Long MD, Rao SS. ACG clinical guideline: small intestinal bacterial overgrowth. Am J Gastroenterol. 2020;115 (2):165-178. doi:10.14309/ajg.0000000000000501

46. Bouras EP, Camilleri M, Burton DD, Thomforde G, McKinzie S, Zinsmeister AR. Prucalopride accelerates gastrointestinal and colonic transit in patients with constipation without a rectal evacuation disorder. Gastroenterology. 2001;120(2):354-360. doi:10.1053/ gast.2001.21166
47. Corsetti M, Thys A, Harris A, et al. High-resolution manometry reveals different effect of polyethylene glycol, bisacodyl, and prucalopride on colonic motility in healthy subjects: an acute, open label, randomized, crossover, reader-blinded study with potential clinical implications. Neurogastroenterol Motil. 2020:e14040.

48. Carbone F, Vanuytsel T, Tack J. The effect of prucalopride on gastric sensorimotor function and satiation in healthy volunteers. Neurogastroenterol Motil. 2021:e14083.

49. Carbone F, Van den Houte K, Clevers E, et al. Prucalopride in gastroparesis: a randomized placebo-controlled crossover study. $\mathrm{Am}$ J Gastroenterol. 2019;114(8):1265-1274. doi:10.14309/ ajg.0000000000000304

50. Andrews CN, Woo M, Buresi M, et al. Prucalopride in diabetic and connective tissue disease-related gastroparesis: randomized placebocontrolled crossover pilot trial. Neurogastroenterol Motil. 2021;33 (1):e13958. doi:10.1111/nmo.13958

51. Emmanuel A, Kamm M, Roy A, Kerstens R, Vandeplassche L. Randomised clinical trial: the efficacy of prucalopride in patients with chronic intestinal pseudo-obstruction-a double-blind, placebo-controlled, cross-over, multiple $\mathrm{n}=1$ study. Aliment Pharmacol Ther. 2012;35(1):48-55. doi:10.1111/j.13652036.2011.04907.x

52. Keefer L, Drossman DA, Guthrie E, et al. Centrally mediated disorders of gastrointestinal pain. Gastroenterology. 2016;150 (6):1408-1419. doi:10.1053/j.gastro.2016.02.034

53. Kilgallon E, Vasant DH, Green D, et al. Chronic continuous abdominal pain: evaluation of diagnostic features, iatrogenesis and drug treatments in a cohort of 103 patients. Aliment Pharmacol Ther. 2019;49(10):1282-1292. doi:10.1111/apt.15241

54. Sobin WH, Heinrich TW, Drossman DA. Central neuromodulators for treating functional GI disorders: a primer. Am J Gastroenterol. 2017;112(5):693-702. doi:10.1038/ajg.2017.57

55. Drossman DA, Tack J, Ford AC, Szigethy E, Tornblom H, Van Oudenhove L. Neuromodulators for functional gastrointestinal disorders (Disorders of Gut-Brain Interaction): a Rome Foundation Working Team Report. Gastroenterology. 2018;154(4):1140-1171 e1141. doi:10.1053/j.gastro.2017.11.279

56. Perlemuter G, Cacoub P, Chaussade S, Wechsler B, Couturier D, Piette JC. Octreotide treatment of chronic intestinal pseudoobstruction secondary to connective tissue diseases. Arthritis Rheum. 1999;42(7):1545-1549. doi:10.1002/1529-0131(199907)42:7<1545:: AID-ANR30>3.0.CO;2-T

57. Soudah HC, Hasler WL, Owyang C. Effect of octreotide on intestinal motility and bacterial overgrowth in scleroderma. $N$ Engl $\mathrm{J} \mathrm{Med.}$ 1991;325(21):1461-1467. doi:10.1056/NEJM199111213252102

58. Ambartsumyan L, Flores A, Nurko S, Rodriguez L. Utility of octreotide in advancing enteral feeds in children with chronic intestinal pseudo-obstruction. Pediatric Drugs. 2016;18(5):387-392. doi:10.1007/s40272-016-0189-x

59. Bharucha AE, Camilleri M, Burton D, Low PA, Gehrking TL, Zinsmeister AR. Pilot study of pyridostigmine in constipated patients with autonomic neuropathy. Clin Auton Res. 2008;18(4):194-202. doi:10.1007/s10286-008-0476-x

60. O'Dea CJ, Brookes JH, Wattchow DA. The efficacy of treatment of patients with severe constipation or recurrent pseudo-obstruction with pyridostigmine. Colorectal Dis. 2010;12(6):540-548. doi:10.1111/ j.1463-1318.2009.01838.x

61. Bharucha AE, Low P, Camilleri M, et al. A randomised controlled study of the effect of cholinesterase inhibition on colon function in patients with diabetes mellitus and constipation. Gut. 2013;62(5):708. doi:10.1136/gutjnl-2012-302483

62. Ahuja NK, Mische L, Clarke JO, Wigley FM, McMahan ZH Pyridostigmine for the treatment of gastrointestinal symptoms in systemic sclerosis. Paper presented at: Seminars in arthritis and rheumatism; 2018 
63. Paine P, McMahon M, Farrer K, Overshott R, Lal S. Jejunal feeding: when is it the right thing to do? Frontline Gastroenterol. 2020;11 (5):397-403. doi:10.1136/flgastro-2019-101181

64. Twist K, Ablett J, Wearden A, et al. Gastrointestinal dysmotility: a qualitative exploration of the journey from symptom onset to diagnosis. Neurogastroenterol Motil. 2018;30(8):e13339. doi:10.1111/nmo.13339

65. Ablett J, Vasant DH, Taylor M, Cawley C, Lal S. Poor social support and unemployment are associated with negative affect in home parenteral nutrition-dependent patients with chronic intestinal failure. J Parenter Enteral Nutr. 2019;43(4):534-539. doi:10.1002/jpen.1457

66. Amiot A, Joly F, Alves A, Panis Y, Bouhnik Y, Messing B. Longterm outcome of chronic intestinal pseudo-obstruction adult patients requiring home parenteral nutrition. Am J Gastroenterol. 2009;104 (5):1262-1270. doi:10.1038/ajg.2009.58

67. Salazar E, Clermont-Dejean NM, Schwenger KJ, et al. Patients with severe gastrointestinal dysmotility disorders on home parenteral nutrition have similar survival as those with short bowel syndrome: a Prospective Cohort Study. J Parenter Enteral Nutr. 2020. doi:10.1002/jpen.1866

68. Appleby R, Yu C, Nightingale J. PTU-106 improved survival and independance from parental nutrition for patients with intestinal dysmotility. Gut. 2016;65(Suppl 1):A107-A107. doi:10.1136/gutjnl2016-312388.192

69. Stanghellini V, Cogliandro RF, De Giorgio R, et al. Natural history of chronic idiopathic intestinal pseudo-obstruction in adults: a single center study. Clin Gastroenterol Hepatol. 2005;3(5):449-458. doi:10.1016/S1542-3565(04)00675-5
70. Murr MM, Sarr MG, Camilleri M. The surgeon's role in the treatment of chronic intestinal pseudoobstruction. Am J Gastroenterol. 1995;90 (12).

71. Mughal M, Irving M. Treatment of end stage chronic intestinal pseudo-obstruction by subtotal enterectomy and home parenteral nutrition. Gut. 1988;29(11):1613-1617. doi:10.1136/gut.29.11.1613

72. Sogawa H, Costa G, Armanyous S, et al. Twenty years of Gut transplantation for chronic intestinal pseudo-obstruction: technical innovation, long-term outcome, quality of life, and disease recurrence. Ann Surg. 2021;273(2):325-333. doi:10.1097/ SLA.0000000000003265

73. Szigethy E, Knisely M, Drossman D. Opioid misuse in gastroenterology and non-opioid management of abdominal pain. Nat Rev Gastroenterol Hepatol. 2018;15(3):168. doi:10.1038/ nrgastro.2017.141

74. Keefer L, Palsson OS, Pandolfino JE. Best practice update: incorporating psychogastroenterology into management of digestive disorders. Gastroenterology. 2018;154(5):1249-1257. doi:10.1053/j. gastro.2018.01.045

75. Riehl ME, Kinnucan JA, Chey WD, Stidham RW. Nuances of the psychogastroenterology patient: a predictive model for gastrointestinal quality of life improvement. Neurogastroenterol Motil. 2019;31 (9):e13663. doi:10.1111/nmo.13663

76. Vasant DH, Whorwell PJ. Gut-focused hypnotherapy for functional gastrointestinal disorders: evidence-base, practical aspects, and the Manchester protocol. Neurogastroenterol Motil. 2019;31(8):e13573. doi:10.1111/nmo.13573
Clinical and Experimental Gastroenterology

\section{Publish your work in this journal}

Clinical and Experimental Gastroenterology is an international, peerreviewed, open access, online journal publishing original research, reports, editorials, reviews and commentaries on all aspects of gastroenterology in the clinic and laboratory. This journal is indexed on American Chemical Society's Chemical Abstracts Service (CAS).

\section{Dovepress}

The manuscript management system is completely online and includes a very quick and fair peer-review system, which is all easy to use. Visit http://www.dovepress.com/testimonials.php to read real quotes from published authors. 